\title{
Characterizing Electrocardiographic Changes During Pre-Seizure Periods Through Temporal and Spectral Features
}

\author{
Lucia Billeci $^{1}$, Maurizio Varanini ${ }^{1}$ \\ ${ }^{1}$ Institute of Clinical Physiology, National Research Council of Italy (IFC-CNR), Pisa, Italy
}

\begin{abstract}
Epilepsy affects the autonomic nervous system, and changes in this function are known to occur during, and even before the electroencephalographic onset of the seizure. The aim of this study was to characterize autonomic changes during and before the epileptic seizures.

The electrocardiographic signals from thirteen epileptic patients, were first pre-processed and then the $R R$ series was extracted. The following segments were selected for the analysis: $15 \mathrm{~min}$ before seizure onset (preictal), seizure time frame (ictal) and $15 \mathrm{~min}$ far from the seizure (interictal). Temporal and frequency features were calculated. In addition, Recurrence Quantification Analysis (RQA) was performed.

Significant differences were detected in time-domain and RQA parameters 15 min before seizures suggesting the possibility of an early prediction of seizure onset. In addition, significant changes were observed during seizure. Further studies are needed to confirm these preliminary results in a larger number of subjects.
\end{abstract}

\section{Introduction}

Epilepsy is a neurological disorder [1], characterized by the recurrence of epileptic seizures, which constitutes a nosographic entity with considerable social impact, both because of its high incidence and of its chronicity. The prevalence of the disorder is estimated at around $1 \%$ of the population. Of these subjects, $25 \%$ do not respond to available therapies [2]. An early prediction of epileptic seizures would considerably increase the quality of life of these patients.

Epileptic seizures can affect autonomic nervous system (ANS) determining changes in both the sympathetic and parasympathetic functions. A recent meta-analysis has been published reporting autonomic changes in epilepsy [3]. Tachycardia and bradycardia are well-known autonomic phenomena associated with epileptic seizures, and such cardiac changes occur not only at the same time as but also prior to the electroencephalographic (EEG) seizure onset $[4,5]$.

The activation of central ANS by epileptic discharge propagation during a seizure is thought to be responsible for the preictal cardiac autonomic symptoms [6]. Few studies have been published characterizing ANS changes during preictal phases with contradictory results [7-9].

The aim of this study was to characterize ANS changes during the seizures and in particular prior to the onset of the seizures, i.e., during the preictal phase. In addition to time and frequency domain parameters, Recurrence Quantification Analysis (RQA) was also applied, for the first time, to characterize ANS during epilepsy. The longterm goal of the study is to develop an algorithm able to predict epileptic seizures with high sensitivity and specificity.

\section{Methods}

\subsection{Data}

Seizures were selected retrospectively from patients recruited at Unit of Neurology and Neurophysiology, Department of Neurological and Neurosensorial Sciences, University of Siena, Italy. All the patients were long-term monitored with 10-20 EEG and ECG. The onset of seizures was annotated based on EEG and video. ECG was measured simultaneously with a sampling rate of $512 \mathrm{~Hz}$.

A total number of 31 seizures were collected from 13 patients affected by various kinds of epilepsy.

\subsection{Pre-processing}

ECG signals were first analysed for impulsive artefacts removal, power-line interference cancelling $(50 \mathrm{~Hz})$, baseline wandering removal, signal-to-noise ratio improvement [10]. The signal was then interpolated to $1024 \mathrm{KHz}$ and the QRS complexes were detected to reconstruct the RR series. Afterwards, an algorithm was applied for the recognition and correction of nonsinusoidal beats in order to have a RR series that only contains variations due to the sinus node and thus reflects the activity of the ANS.

\subsection{Feature extraction}

For each seizure, three ECG segments were selected:

- interictal $15 \mathrm{~min}$ epoch apart from at least 50 minutes from seizure onset; 
- preictal 15 min epoch ending $30 \mathrm{~s}$ before the seizure onset;

- ictal epoch during seizure.

For each segment, features were extracted within 1-min non-overlapping windows. To reduce inter-individual variability, the RR was normalized with zero mean and a standard deviation of one before computing the features.

\subsection{Time-domain features}

The time-domain features were the mean of $R R$ intervals (MeanNN); the number of pairs of adjacent RRI whose difference is more than $50 \mathrm{~ms}$ (NN50); the standard deviation of projection of the Poincaré plot on the line perpendicular to the line of identity that is a measure of short-term variability (SD1); the standard deviation of the projection of the Poincare plot on the line of identity that is a measure of long-term variability (SD2); the Cardiac Sympathetic Index (CSI=SD2/SD1).

\subsection{Frequency-domain features}

To compute frequency-domain features, the Generalized Short Time Fourier Transform (GSTFT) [11] was calculated. From the GSTFT we extracted the power of the low frequency band $(0.04 \mathrm{~Hz}-0.15 \mathrm{~Hz})$ normalized to the total power (LFn), the power of the high frequency band $(0.15 \mathrm{~Hz}-0.40 \mathrm{~Hz})$ normalized to the total power (HFn) and the ratio of LF to HF (LF/HF), which is related to the sympathetic-parasympathetic balance of the ANS.

\subsection{Recurrence quantification analysis}

RQA [12] quantifies the density of recurrence points as well as the histograms of the lengths of the diagonal and vertical lines in a recurrence plot. Parameters extracted were the Recurrence Rate (RR), Determinism (DET), Laminarity (LAM), Entropy (ENT), the maximum length of the diagonal (Lmax) and the Trapping Time (TT).

\subsection{Statistical analysis}

Statistical analyses were performed using IBM SPSS 20 for Mac (IBM, Armonk, NY, USA). The Shapiro-Wilk test was applied to test normality of variables. As the data were asymmetrically distributed, the Friedman test for repeated measures was used to compare features in preictal, ictal and interictal segments. If it showed a significant effect, then a paired Wilcoxon test for post-hoc analyses was applied. Values are given as median(IQR) and a $p<0.05$ was considered as statistically significant.

\section{Results}

\subsection{Time-domain analysis}

Table 1 reports time-domain features in interictal, preictal and ictal segments with significance. In particular, we observed that NN50 was significantly different in the three different epochs as shown in Figure 1.

Table 1. Time-domain features in interictal, preictal and ictal segments.

\begin{tabular}{|c|c|c|c|}
\hline Feature & Value & $\begin{array}{l}\text { Friedman } \\
\text { Test } \\
\text { (p-value) }\end{array}$ & $\begin{array}{l}\text { Wilcoxon } \\
\text { Test }{ }^{\S} \\
\text { (p-value) }\end{array}$ \\
\hline MeanNN & & 0.51 & \\
\hline Interictal & $0.75(0.09-3.16)$ & & \\
\hline Preictal & $0.22(-0.55-4.73)$ & & \\
\hline Ictal & 8.98(-1.96-17.87) & & \\
\hline NN50 & & $0.019^{*}$ & \\
\hline Interictal & $43.22(17.82-47.51)$ & & \\
\hline Preictal & $32.01(14.79-48.08)$ & & $0.03 *$ \\
\hline Ictal & $19.26(14.91-38.56)$ & & $0.005 * *$ \\
\hline$S D 1$ & & $0.02 *$ & \\
\hline Interictal & $0.17(0.09-0.25)$ & & \\
\hline Preictal & $0.16(0.07-0.27)$ & & 0.91 \\
\hline Ictal & $0.94(0.18-15.56)$ & & $0.001 * *$ \\
\hline$S D 2$ & & $<0.001 * *$ & \\
\hline Interictal & $0.52(0.37-1.21)$ & & \\
\hline Preictal & $0.51(0.32-0.77)$ & & 0.87 \\
\hline Ictal & $2.00(0.92-15.56)$ & & $<0.001 * *$ \\
\hline CSI & & $<0.001 * *$ & \\
\hline Interictal & $3.59(2.83-4.06)$ & & \\
\hline Preictal & $3.22(2.84-5.13)$ & & 0.48 \\
\hline Ictal & $8.52(6.44-15.56)$ & & $<0.001 * *$ \\
\hline
\end{tabular}

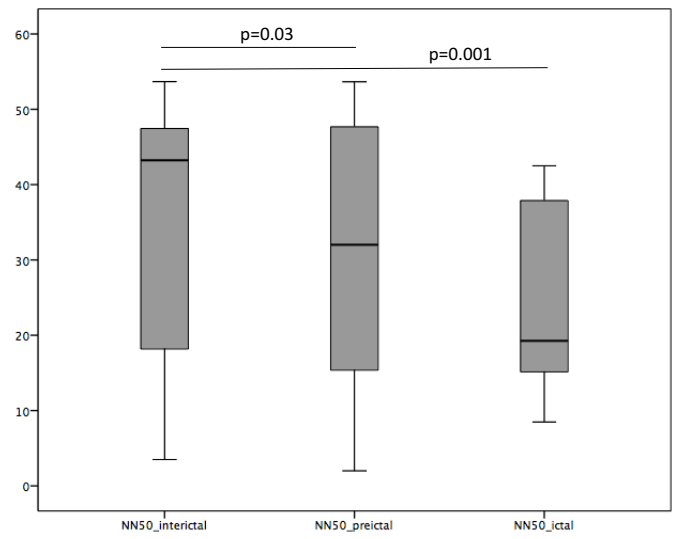

Figure 1. NN50 in the different epochs. ${ }^{*} \mathrm{p}<0.05,{ }^{*} \mathrm{p}<0.01$ 


\subsection{Frequency-domain analysis}

Table 2 reports frequency-domain features in the different phases with significance. LF/HF was significantly increased during ictal compared to interictal.

Table 2. Frequency-domain features in interictal, preictal and ictal segments.

\begin{tabular}{llll}
\hline Feature & Value & $\begin{array}{l}\text { Friedman } \\
\text { Test } \\
\text { (p-value) }\end{array}$ & $\begin{array}{l}\text { Wilcoxon } \\
\text { Test }^{\S} \\
(\mathrm{p} \text {-value })\end{array}$ \\
\hline$L F n$ & & 0.96 & \\
Interictal & $0.22(0.18-0.27)$ & & \\
Preictal & $0.23(0.18-0.28)$ & & \\
Ictal & $0.17(0.11-15.56)$ & & \\
$H F n$ & & 0.40 & \\
Interictal & $0.17(0.12-0.27)$ & & \\
Preictal & $0.13(0.08-0.25)$ & & \\
Ictal & $0.17(0.05-15.56)$ & & \\
LF/HF & & $0.03^{*}$ & \\
Interictal & $2.19(1.53-4.04)$ & & 0.12 \\
Preictal & $2.84(1.62-4.57)$ & & $0.007^{* *}$ \\
Ictal & $4.37(2.84-15.56)$ & & \\
\hline
\end{tabular}

${ }^{\S}$ Comparisons to interictal, reported only when the Friedman test was significant. ${ }^{*} p<0.05,{ }^{* *} p<0.01$

\subsection{Recurrence quantification analysis}

Results of the RQA analysis with significance are reported in Table 3.

Table 3. RQA parameters in interictal, preictal and ictal segments.

\begin{tabular}{|c|c|c|c|}
\hline Feature & Value & $\begin{array}{l}\text { Friedman } \\
\text { Test } \\
\text { (p-value) }\end{array}$ & $\begin{array}{l}\text { Wilcoxon } \\
\text { Test }{ }^{\S} \\
\text { (p-value) }\end{array}$ \\
\hline$R R$ & & 0.96 & \\
\hline Interictal & $13.05(7.62-31.23)$ & & \\
\hline Preictal & $15.75(7.86-65.24)$ & & \\
\hline Ictal & $18.63(10.88-28.89)$ & & \\
\hline DET & & 0.43 & \\
\hline Interictal & $57.58(17.82-77.66)$ & & \\
\hline Preictal & $63.83(14.79-86.91)$ & & \\
\hline Ictal & $19.25(13.62-63.27)$ & & \\
\hline$L A M$ & & 0.30 & \\
\hline Interictal & $18.87(8.98-44.23)$ & & \\
\hline Preictal & $16.49(7.17-70.09)$ & & \\
\hline Ictal & $19.25(13.26-49.60)$ & & \\
\hline ENT & & $0.001 * *$ & \\
\hline Interictal & $2.36(1.38-3.23)$ & & \\
\hline
\end{tabular}

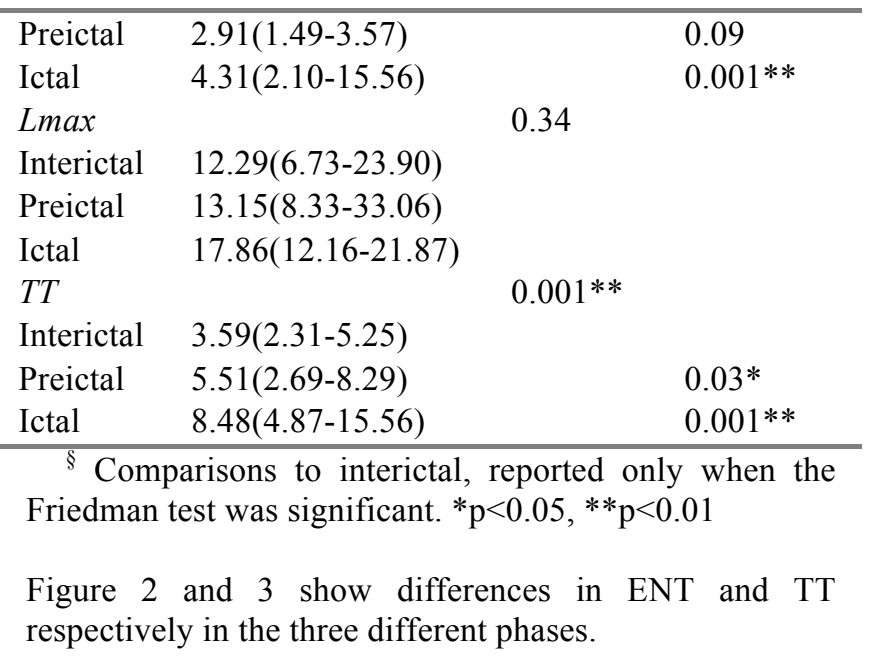

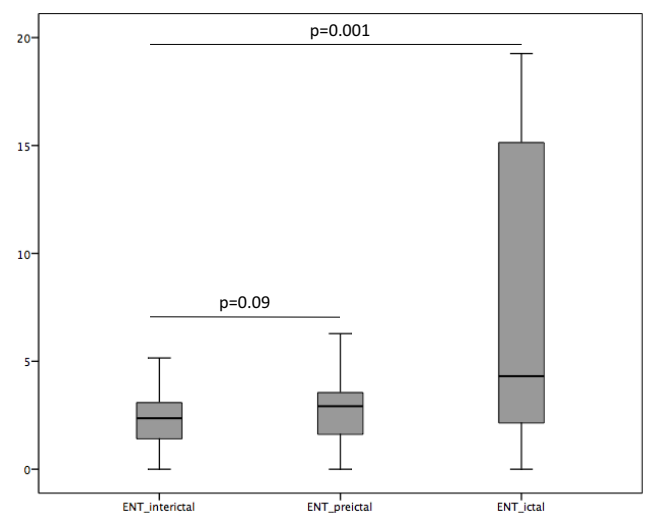

Figure 2. ENT in the different epochs. ${ }^{*} p<0.05,{ }^{* *} p<0.01$

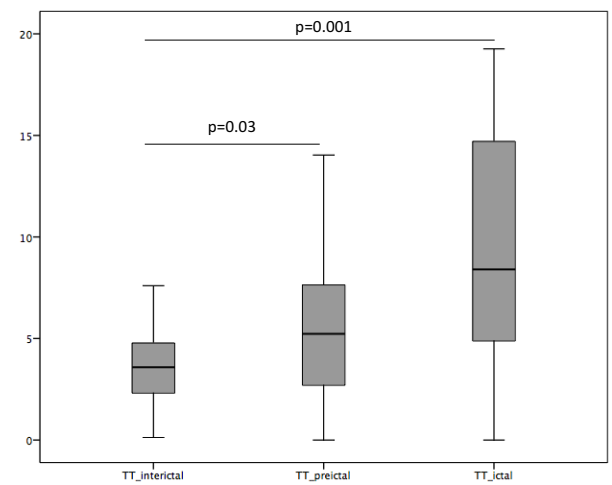

Figure 3. TT in the different epochs. ${ }^{*} \mathrm{p}<0.05,{ }^{* *} \mathrm{p}<0.01$

\section{Discussion}

The most significant result of our study was the significant changes in preictal phase compared to the interictal phase, suggesting that it is possible to predict seizures.

In particular, we observed a decrease in NN50, suggesting a deterioration of HRV, which could be an 
indication of increased cardiovascular risk, including mortality [13]. Significantly decreased in NN50 was previously reported during epilepsy [14]. In addition, it was recently observed, using a KNN classifier, that NN50 and pNN50 were the most relevant features for predicting epileptic seizures [15]. We also observed differences in RQA parameters in preictal phase that was for the first time applied for the characterization of ANS during seizures. In particular, an increase in ENT and TT in preictal compared to interictal phase was observed, although for ENT in preictal there was a marginally significance. The increase in ENT means greater complexity of the recurrence plots during seizures. The increase in TT means that the time that the system abides at a specific state during seizures is longer than interictal phase. This change, previously observed applying RQA analysis to EEG signals in epilepsy [16], could reflect the synchronization of neurons during seizures.

Other findings of this study are specific to ictal phase. In particular, we observed an increase in SD1, SD2, CSI and LF/HF. Overall these results suggest a predominance of sympathetic activity during seizure. Our finding confirms previous studies, which reported significant increase in several indices of sympathetic function before and during seizures [8], [14, 17]. These results indicate a sudden and excessive sympathetic shift in the sympathovagal balance of ANS before the seizure-onset.

Overall the findings of this study suggest significant changes in ANS at least 15 min before seizures. Notably, the preictal phase selected in our study terminated $30 \mathrm{~s}$ before seizure, a sufficient time before the seizure onset to give an alarm to the subject. Thus, the results of this study could help in the prediction of the seizures to prevent adverse effects and in the automatic detection of seizures. Further studies are needed to confirm these preliminary results in a larger number of subjects. In addition, in a larger sample, seizures could be differentiated according to their localization to for a better characterization.

\section{Acknowledgements}

This study was supported by Bando FAS Salute Sviluppo Toscana 2014-PANACEE Project (No. 6247).

\section{References}

[1] Tatum WO, Kaplan PW, Jallon P, Epilepsy, a Concise Encyclopedia. Demos Medical Publishing, 2009.

[2] Epilepsy Facts and Figures. Landover. Epilepsy Foundation of America, 1999.

[3] Lotufo PA, Valiengo L, Benseñor IM, Brunoni AR. A systematic review and meta-analysis of heart rate variability in epilepsy and antiepileptic drugs. Epilepsia 2012;53:272-82.

[4] Gennaro GD, Quarato PP, Sebastiano F, Esposito V, Onorati P, Grammaldo LG, Meldolesi GN, Mascia A, Falco
C, Scoppetta C, Eusebi F, Manfredi M, Cantore G, Ictal heart rate increase precedes EEG discharge in drugresistant mesial temporal lobe seizures. Clin Neurophysiol 2004;115:1169-1177.

[5] Kato K, Jin K, Itabashi H, Iwasaki M, Kakisaka Y, Aoki M, Nakasato N. Earlier tachycardia onset in right than left mesial temporal lobe. Neurology 2014;83,1332-1336.

[6] Jansen K, Varon C, Van Huffel S, Lagae L. Peri-ictal ECG changes in childhood epilepsy: implications for detection systems. Epilepsy Behav 2013;29:72-6.

[7] Toth V, Hejjel L, Fogarasi A, Gyimesi C, Orsi G, Szucs A, Kovacs N, Komoly S, Ebner A, Janszky J. Periictal heart rate variability analysis suggests long-term postictal autonomic disturbance in epilepsy. Eur $\mathrm{J}$ Neurol 2010;17:780-7.

[8] Behbahani S, Dabanloo NJ, Nasrabadi AM, Teixeira CA, Dourado A. Preictal heart rate variability assessment of epileptic seizures by means of linear and non-linear analyses. Anadolu Kardiyol Derg 2013;13:797-803.

[9] Jansen K, Varon C, Van Huffel S, Lagae L. Peri-ictal ECG changes in childhood epilepsy: implications for detection systems. Epilepsy Behav 2013;29:72-6.

[10] Varanini M, Tartarisco G, Billeci L, Macerata A, Pioggia $\mathrm{G}$, Balocchi R. An efficient unsupervised fetal QRS complex detection from abdominal maternal ECG. Physiol Meas 2014; 35:1607- 19.

[11] Varanini M, De Paolis G, Emdin M, Macerata A, Pola S, Cipriani M, Marchesi C. A Multiresolution Transform for the Analysis of Cardiovascular Time Series, Computers in Cardiology, IEEE Computer Society Press, 1998.

[12] Webber Jr. CL, Zbilut JP. Dynamical assessment of physiological systems and states using recurrence plot strategies. Journal of Applied Physiology 1994;76:965973.

[13] May O, Arildsen H. Long-term predictive power of heart rate variability on all-cause mortality in the diabetic population. Acta Diabetol 2011;48:55-59.

[14] Kolsal E, Serdaroğlu A, Cilsal E, Kula S, Soysal AŞ, Kurt AN, Arhan E. Can heart rate variability in children with epilepsy be used to predict seizures? Seizure 2014;23:35762.

[15] Hoyos-Osorio K., Castañeda-Gonzaiez J. and DazaSantacoloma G. Automatic epileptic seizure prediction based on scalp EEG and ECG signals, 2016 XXI Symposium on Signal Processing, Images and Artificial Vision (STSIVA), Bucaramanga, 2016, pp. 1-7.

[16] Ng EEP, Lim NG, Chattopadhyay S, Muralidhar B. Automated Identification of Epileptic and Alcoholic EEG Signals using Recurrence Quantification Analysis. Journal of Mechanics in Medicine and Biology 2012;12.

[17] Jeppesen J, Beniczky S, Johansen P, Sidenius P, FuglsangFrederiksen A. Detection of epileptic seizures with a modified heart rate variability algorithm based on Lorenz plot. Seizure 2015;24:1-7.

Address for correspondence.

Lucia Billeci

Institute of Clinical Physiology, National Research Council of Italy (CNR), via Moruzzi 1, 56127, Pisa, Italy

lucia.billeci@ifc.cnr.it 